\title{
Case Files of the Children's Hospital of Michigan Regional Poison Control Center: The Use of Carnitine for the Management of Acute Valproic Acid Toxicity
}

\author{
Abhishek Katiyar, $M D^{a}$, Cynthia Aaron, $M D^{a}$ \\ aChildren's Hospital of Michigan Regional Poison Control Center, Detroit, MI
}

\section{CASE PRESENTATION}

A 28-year-old African-American male was found unresponsive outside of his girlfriend's house around 7 o'clock in the morning. He spent the night at the girlfriend's house and was last witnessed to be awake about 12 hours earlier. The girlfriend and her family were unable to awaken the patient and called emergency medical services (EMS). There was no history of headache, dizziness, fever, chills, nausea, vomiting, seizures, trauma, depressive thoughts, or suicidal ideation. When EMS arrived, they administered $6 \mathrm{mg}$ of naloxone intramuscularly without effect, and the patient was transported to the emergency department (ED).

After arriving in the ED, the cardiac monitor displayed a narrow-complex rhythm with a rate of 85-90 per minute. His initial vital signs were: heart rate $91 / \mathrm{min}$; blood pressure $148 / 80$ $\mathrm{mmHg}$; respiratory rate $24 / \mathrm{min}$; temperature $35.1^{\circ} \mathrm{C}\left(95.1^{\circ} \mathrm{F}\right)$ rectally; and pulse oximetry $100 \%$ on non-rebreather face mask oxygen. A fingerstick glucose determination was $106 \mathrm{mg} / \mathrm{dL}$.

Physical examination revealed a well developed, well nourished individual who was obtunded and unresponsive. His pupils were $2 \mathrm{~mm}$, unreactive to light, and without nystagmus. He had diffuse bilateral wheezing, regular heart rate without any murmurs, and decreased bowel sounds. The patient had warm skin with good distal pulses in all extremities and no cyanosis, erythema, or edema. There was no clonus or hyperreflexia, and the gag reflex was intact.

His past medical history was only pertinent for an old gunshot wound to the head. He developed a seizure disorder and was taking divalproex, $500 \mathrm{mg}$ BID. Reportedly, he was allergic to carbamazepine and phenytoin. The patient smoked cigarettes, but the family stated that he had no history of alcohol or illicit drug use.
A 12-lead electrocardiogram showed a sinus rhythm with a rate of $92 \mathrm{bpm}$, a PR interval of $134 \mathrm{msec}$, a QRS duration of $90 \mathrm{msec}$, and a QTc of $601 \mathrm{msec}$. A head CT and chest radiograph were unremarkable. Laboratory tests were obtained.

\section{What is Valproic Acid?}

Valproic acid (VPA) is an antiepileptic drug commonly prescribed worldwide for various neurological and psychological conditions. It was first synthesized by Burton in 1882 as a clear, colorless, fatty acid liquid. For eight decades, it was used as an organic solvent in research laboratories until it was noted to have anticonvulsant effects in rodents [1]. By 1967, it was approved as a therapy for generalized seizures in France, and in 1983 it was approved by the United States Food and Drug Administration (FDA) [1]. A decade later, divalproex sodium (Depakote ${ }^{\circledR}$ )—essentially a dimer of VPA - was synthesized and marketed as a longer acting formulation than VPA alone [1]. At the same time, the FDA approved valproic acid for use in the management of bipolar disorder. It is currently indicated for the treatment for generalized and focal epilepsy, migraine headaches, bipolar disorder, trigeminal neuralgia, and other neuropathic pain $[2,3]$. The drug is commercially available in several forms that include extended-release tablets, uncoated tablets, enteric-coated tablets, liquid elixir formula, soft-gelatin capsules, sprinkle capsules, and an intravenous formulation. The liquid formulation can be administered orally or rectally $[3,4]$.

Recently, there has been renewed interest in the use of VPA for the treatment of HIV infection and various cancers. Valproate is believed to be an inhibitor of the enzyme histone deacetylase 1 (HDAC1) [5-7]. This enzyme is responsible for the integrity and survival of HIV infected $\mathrm{CD} 4^{+} \mathrm{T}$ cells. A study published in

Keywords: valproic acid, valproate, carnitine

Notes: There was no outside funding of any kind used for this study.

Corresponding Author: Abhishek Katiyar, MD, 4616 John R, Suite 616, Detroit, MI 48201. Email: akatiyar2@dmc.org 
August 2005 revealed that VPA, in combination with antiretroviral drugs, produced a $75 \%$ reduction in HIV infection [5]. Furthermore, by inhibiting the HDAC1 enzyme, VPA is cytotoxic to many different cancer types, including multiple myeloma and melanoma [6,7]

The increased use and availability of VPA have led to a steady increase in the number of cases reported to the American Association of Poison Control Centers' (AAPCC) Toxic Exposure Surveillance System (TESS) [8]. From 1995 to 2000, there was a $129 \%$ increase in the number of VPA-exposures reported to the AAPCC, a 707\% rise since 1990 [9-11]. In 2000, data from the TESS database reported 5204 intentional VPA exposures and 3880 unintentional exposures; of these exposures, 373 resulted in major toxicity and 16 resulted in deaths $[8,11]$.

\section{How does VPA work as an antiepileptic?}

VPA's exact mechanism of action is not fully understood, but there are several proposed theories. First, valproate is believed to work by increasing levels of the inhibitory neurotransmitter $\gamma$-aminobutryic acid (GABA) in the central nervous system (CNS). In multiple studies, acute administration of VPA increases GABA concentrations in the animal brain models by 15 to $45 \%[12,13]$. This is accomplished by inhibiting the enzymes GABA transminase and succinate semialdehyde dehydrogenase; both responsible for the breakdown of GABA [8]. It is also thought that VPA suppresses N-methyl-D-aspartate (NMDA) activity, which results in attenuation of neuronal excitation. By reducing excitatory synaptic transmission, VPA decreases synchronized cell firing which leads to decreased epileptic bursts [14-16]. Valproic acid may also reduce repetitive firing of neurons through direct effects on neuronal membranes by altering sodium and potassium conductance $[3,4,8,17]$. However, recent studies on rat hippocampal sections have indicated that sodium channel blockade does not appear to be a major contributor $[8,18]$.

\section{Explain the pharmacokinetics of VPA}

Valproic acid is a branched chain carboxylic acid (2-propylpentanoic acid or di-n-propylacetic acid) with a chemical structure very similar to that of short chain fatty acids [19] (Figure 1). Divalproex is a stable complex of equimolar quantities of sodium salt and the valproic acid, which dissociates in the gastrointestinal tract and slowly releases the valproate ion [1]. It is enteric coated and has a slower release in the gastrointestinal tract compared to valproic acid (Depakene ${ }^{\circledR}$ ).

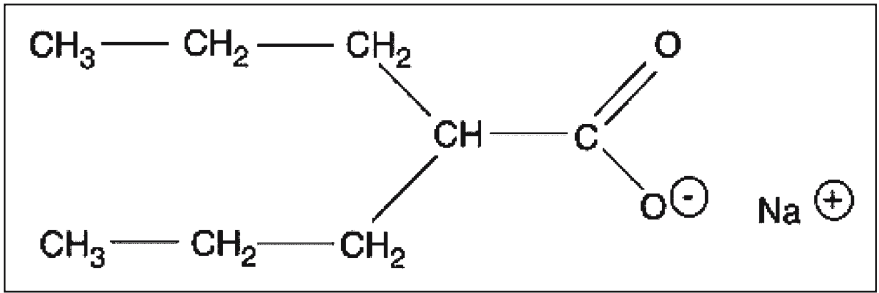

Figure 1: Valproic acid
Non-enteric coated VPA is almost 100\% absorbed from the gastrointestinal tract, reaching a peak serum concentration within 1-4 hours after ingestion [20-22]. Peak plasma concentration for enteric-coated tablets occurs 4-8 hours after ingestion of a therapeutic dose $[4,23]$. In large overdoses or with co-ingestions of other drugs, delayed peak levels have been reported. Graudins and Aaron reported a case of a 32-year-old woman who ingested divalproex sodium and chlorpheniramine and developed a delayed peak serum concentration $17 \mathrm{hrs}$ post-ingestion [23]. Brubacher et al. reported a case of an individual who presented to the ED asymptomatic and hemodynamically stable after ingesting an unknown amount of enteric-coated divalproex sodium. Seven hours later, she developed clinical deterioration from valproate toxicity requiring endotracheal intubation and close monitoring. Her serum level peaked at approximately $13 \mathrm{hrs}$ post ingestion [4].

Approximately $90-95 \%$ of the drug is protein-bound at therapeutic levels [21]. As levels continue to rise, protein binding is saturated, resulting in a large fraction of unbound valproic acid $[24,25]$. Klotz and Antonin reported that when serum VPA concentrations exceeded $150 \mu \mathrm{g} / \mathrm{mL}$, protein binding decreased to about $70 \%$. At serum VPA levels of $300 \mu \mathrm{g} / \mathrm{mL}$, protein binding dropped to $35 \%$ [21]. This phenomenon can also be seen in patients with uremia and low albumin, affecting critical care patients, newborns, elderly, or patients with end-stage renal disease $[21,26,27]$. During elimination, the drug follows first-order Michaelis-Menton kinetics $[8,28,29]$. At therapeutic concentrations, the elimination half-life ranges between 8 to $21.5 \mathrm{hrs}$. In an overdose, the elimination half-life may be prolonged up to $30 \mathrm{hrs}$ or more $[21,25,28]$.

\section{How is valproic acid metabolized?}

In healthy individuals, less than $1-3 \%$ of valproic acid is excreted unchanged in the urine; the rest of the drug undergoes extensive and complex metabolism in the liver [8,19-21,30,31]. It is metabolized in a multi-step process involving direct glucuronidation and followed by either mitochondrial $\beta$-oxidation or cytosolic $\omega$ oxidation to produce multiple metabolites. A total of nine metabolites have been identified, of which the majority are $\beta$-oxidation metabolites: 2-propyl-2-pentenoic acid (2-en-VPA), 3-hydroxy-2propylpentanoic acid (3-OH-VPA), and 3-oxo-2-propylpentanoic acid (3-keto-VPA) $[8,29,31]$. The other six metabolites are produced after undergoing $\omega$-oxidation in the endoplasmic reticulum: 5-hyroxy-2-propylpentanoic acid (5-OH-VPA), 2-polyglutaric acid (PGA), 2-propyl-4-pentenoic acid (4-en-VPA), 4-hydroxy-2propylpentanoic acid (4-OH-VPA), 4-oxo-2-propylpentanoic acid (4-keto-VPA), and 2-propyl-3-pentenoic acid (3-en-VPA) $[8,29,31]$ (Figure 2). Although these latter metabolites have a minor role, they can be highly toxic in patients on chronic therapeutic dosing or after an acute overdose. 4-en-VPA has been incriminated in the development of cerebral edema, hepatotoxicty, and hyperammonemia [8,19,29,31-48].

When metabolized through the mitochondrial $\beta$-oxidation pathway, VPA is transported into the mitochondrial matrix using 


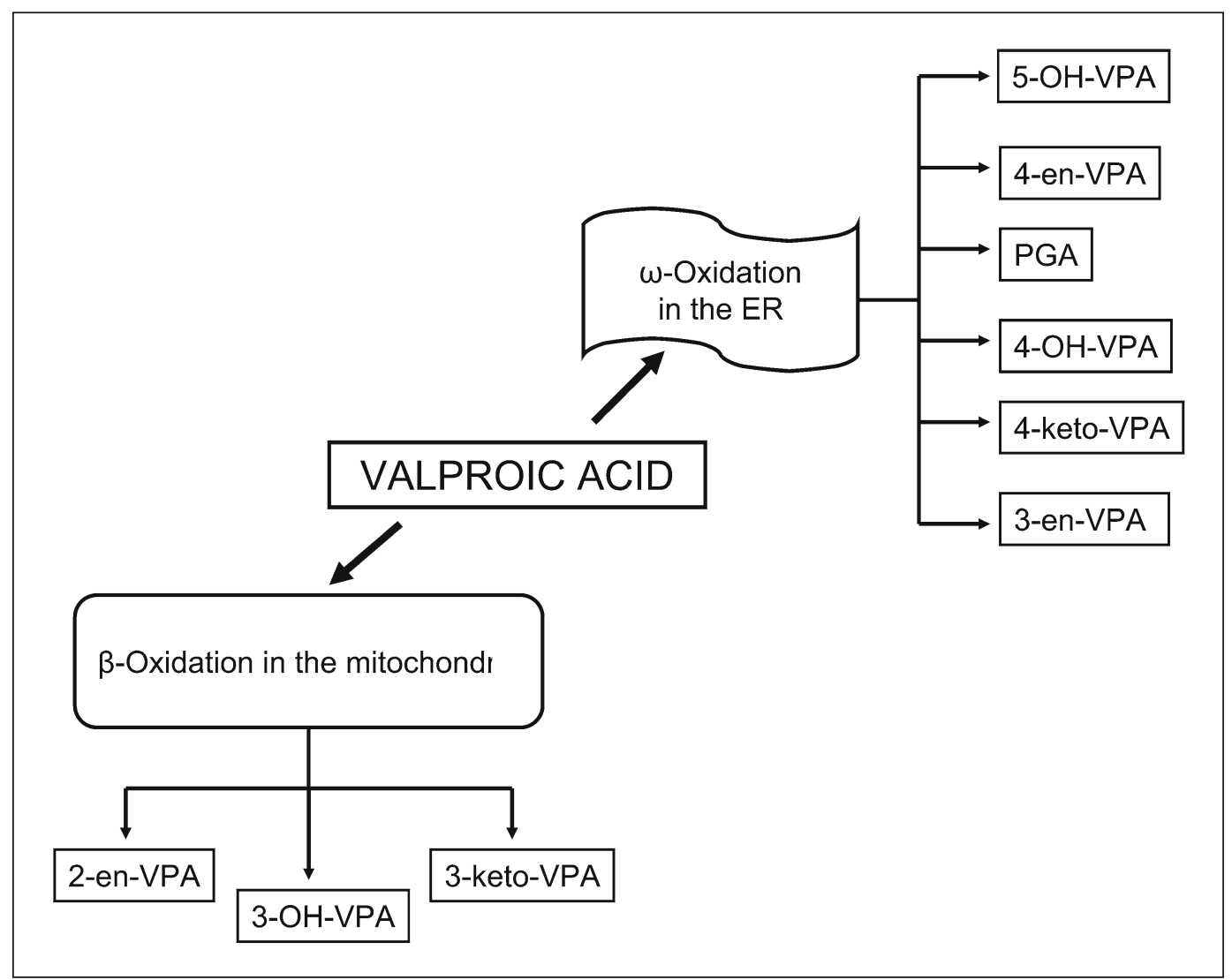

Figure 2: Valproic Acid Metabolism

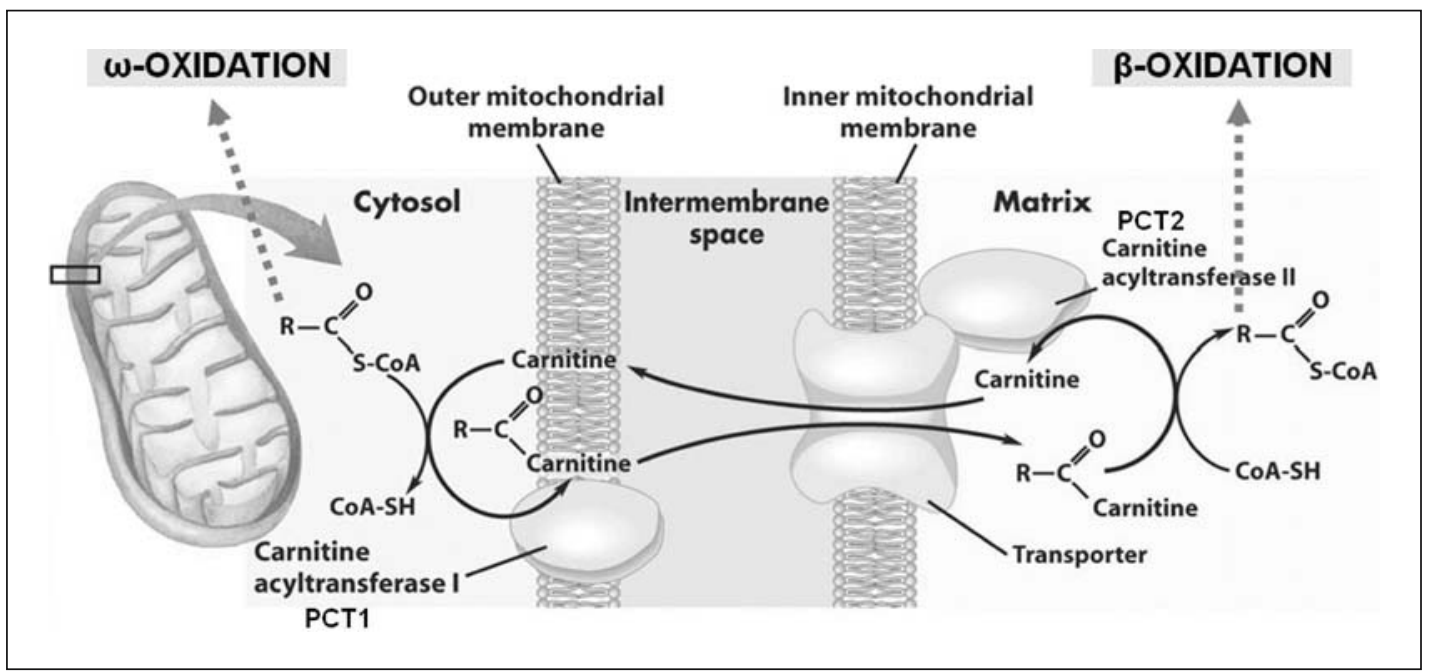

Figure 3: VPA and the Carnitine Shuttle.

Source: Permission granted from Edward M. Marcotte, PhD, University of Texas at Austin

the carnitine shuttle. This is the same pathway involved with the transport of long chain fatty acids. In the cytosol, VPA is linked with reduced acetyl co-enzyme A (CoA-SH) to form valproyl-CoA by an ATP-dependent acyl-CoA synthetase that is located on the outer part of the mitochondrial membrane. This linkage allows valproic acid to cross the outer membrane. Once it passes the outer membrane, valproyl-CoA is then transformed to valproylcarnitine by the palmityl carnitine transferase (PCT1) (Figure 3). This step is necessary since the inner mitochondrial membrane is impermeable to valproyl-CoA. Once inside the mitochondrial 
matrix, the PCT2 transforms valproylcarnitine back to valproylCoA, enabling it to enter the $\beta$-oxidation process $[8,19]$. Hence, carnitine appears to be an essential participant in the metabolism of valproic acid and long chain fatty acids.

\section{What is carnitine?}

Carnitine (Figure 4) is a quaternary amine synthesized from the amino acids lysine and methionine. Carnitine has numerous metabolic functions. It aids in the transport of fatty acids into the mitochondria; it minimizes the toxicity of acyl compounds by modulating the ratio of acetylated coenzyme A to unbound Co-A; and it functions in other cellular metabolism, such as the urea cycle $[49,50]$. Although two enantiomers of carnitine exist, only L-carnitine has biological activity [49].

L-carnitine is an essential co-factor in the $\beta$-oxidation of fatty acids. It catalyzes the transfer of these molecules into the mitochondria where oxidation occurs. Although it serves many biological functions, L-carnitine is not considered an essential nutrient because it is endogenously synthesized [49]. In addition, there is no established recommended daily allowance (RDA) for carnitine by the US FDA. Seventy-five percent of carnitine comes from our diet, particularly in red meat and dairy products. A well balanced diet contains a significant amount of carnitine (20-200 $\mathrm{mg} /$ day for a $70 \mathrm{~kg}$ person). Even strict vegetarian diets contain as much as $1 \mathrm{mg}$ /day (for a $70 \mathrm{~kg}$ person) [19,51]. Pharmaceutically, L-carnitine is available as an oral or parenteral preparation which can be given either intravenously or intramuscularly [19].

Carnitine is also produced endogenously. Humans synthesize approximately 1-2 micromole of carnitine/kg/day [52]. Carnitine synthesis is a multi-step process that occurs in various human tissues including the kidney, liver, heart, muscle, and brain [53]. The final step in the biosynthesis of L-carnitine from lysine involves the conversion of gamma-butyrobetaine to L-carnitine via the enzyme gamma-butyrobetaine hydroxylase [54]. This enzyme is relatively deficient in newborns and young infants. After synthesis, L-carnitine is circulated and taken up by tissues and organs with high metabolic demands such as the myocardium, liver, and adrenal glands [53]. Serum carnitine concentrations represent only about $0.6 \%$ of the total body stores $[19,36,38,40]$.

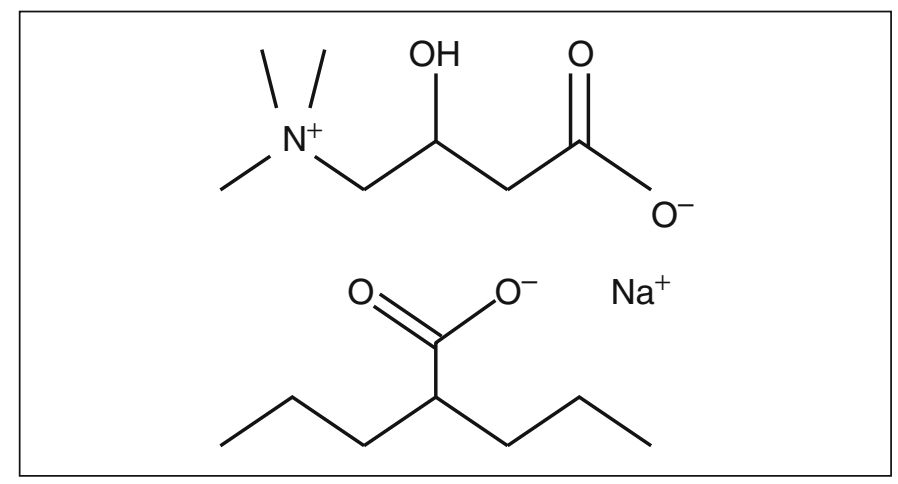

Figure 4: Carnitine
Under some circumstances, the physiological need for carnitine exceeds the biosynthesis production and stores. Specific genetic defects and certain drugs, including valproic acid, can negatively affect human carnitine status [55].

\section{How does valproate cause carnitine deficiency?}

Valproate is known to cause carnitine deficiency; however, the etiology behind this deficiency is subject to debate. It is postulated that long-term administration of valproic acid or high dose therapy may affect carnitine stores by several mechanisms. First, VPA can alter the absorption from food or supplements $[45,50]$. Second, it can reduce the biosynthesis of carnitine from the liver and kidneys by interfering with the mitochondrial CoA-SH and the enzyme gamma-butyrobetaine hydroxylase. When the CoASH is depleted in the mitochondria, free carnitine cannot be regenerated from acylcarnitine. The latter enzyme, butyrobetaine hydroxylase, is responsible for the endogenous synthesis of carnitine [19]. Finally, VPA can also decrease the reabsorption of valproyl-carnitine, acyl-carnitine, and free carnitine from the kidneys $[19,45,50]$. Stadler et al. challenged this theory in an article. In their study, 13 human adult male subjects were administered VPA for nearly 28 days, and plasma and urine samples were measured for free and total carnitine. They concluded that, during long term VPA treatment, there is enhanced reabsorption of carnitine and diminished excretion from the kidneys [45]. Subsequent research supports the theory of valproate impairing the reabsorption of L-carnitine, thereby decreasing serum carnitine levels [56].

\section{So what happens to VPA when carnitine is depleted?}

Carnitine serves two main metabolic functions; it facilitates the transport of valproic acid into the mitochondria, and it prevents the accumulation of valproyl-CoA $[19,57]$. When carnitine is depleted, the transport of valproic acid into the mitochondrial matrix is impaired, decreasing $\beta$-oxidation and shifting the metabolism of VPA towards $\omega$-oxidation in the endoplasmic reticulum. This results in excessive production and accumulation of $\omega$-oxidation metabolites, specifically the hepatotoxic 4-en-VPA metabolite. This metabolite also interferes with the enzymes

\section{Table 1: Risk Factors Associated with Carnitine Deficiency}

Infants and young children

Concomitant neurologic disorders

Congenital metabolic disorders

Liver cirrhosis

Renal Failure

Critical care patients (burns, sepsis, trauma, organ failure, etc)

Use of multiple antiepileptic drugs

Other drugs (chemotherapy agents, anti-nucleoside analogues, etc)

See references: $[19,32,45,47,60,88]$ 
responsible for eliminating ammonia via the urea cycle. Hence, carnitine depletion indirectly blocks the urea cycle resulting in an accumulation of ammonia $[8,19,41]$. Murakami et al. analyzed the urinary metabolites of VPA in a child who was chronically taking VPA for epilepsy. They initially found large amounts of VPA $\omega$-oxidation metabolites, but no metabolites from the $\beta$ oxidation pathway. However, when the patient received exogenous carnitine, significant amounts of $\beta$-oxidation metabolites were detected [40]. This led to the conclusion that carnitine depletion plays a significant role in the metabolism of the intermediary metabolites of VPA. Similar findings were seen in patients with acute VPA intoxication [58].

Decreased carnitine stores can also create an imbalance in the ratio of free CoA to acyl-CoAs, favoring the latter. Accumulation of acyl-CoAs can impair several enzymatic processes, including gluconeogenesis and $\alpha$-ketoacid oxidation and further impairment of $\beta$-oxidation of VPA $[19,41,36]$. Ishikura et al. showed an increase in urinary acyl-CoA after administration of L-carnitine in a 16-month-old child who ingested a massive amount of VPA [36].

\section{What occurs with Valproic Acid toxicity?}

Although side effects from VPA therapy are generally considered mild and transient, serious medical problems (such as hepatatoxicity, hyperammonemia, pancreatitis, and coagulopathy) have occurred $[2,43,44,59]$.

VPA-induced hepatotoxicity results in four distinct patterns which include a transient rise in the liver enzymes, reversible hyperammonemia, fatty infiltration of the liver with resultant hypoglycemia, and progressive hepatic failure [60]. During routine use, VPA may induce elevations in liver enzymes shortly after the initiation of the drug; however, these enzymes almost always return to normal after the reduction of dose or with the supplementation of L-carnitine [32,37]. Any alteration in VPA metabolism, as seen with acute overdose, chronic dosing, or fulminant hepatic failure, will cause an elevation of $\omega$-oxidation products resulting in further hepatic injury [61]. In rat models, the metabolite 4-en-VPA causes microvesicular steatosis in the liver [62]. The concomitant use of phenobarbital therapy with VPA has been shown to induce the cytosolic $\omega$-oxidation pathway and further increase the risk of hepatoxicity $[33,63]$.

Hyperammonemia has been associated with the development of brain edema and subsequent seizures, stupor, and coma [64]. Although the incidence of hyperammonemia associated with hepatic encephalopathy is over 90\% in the United States, elevated serum ammonia can be seen in patients therapeutically taking VPA or after a VPA overdose without any evidence of hepatic injury or failure $[30,42,64,65]$. Duarte et al. reported a case of a 46-year-old woman who developed isolated hyperammonemia without any evidence of hepatic failure after being started on the drug [33]. VPA can cause elevation of serum ammonia by various, distinct pathways. Initially, cerebral edema was thought to be due to the metabolite 2-en-VPA, a product of VPA after $\beta$-oxidation metabolism. Additionally, the metabolites of VPA can interfere with the activity of carbamoyl phosphate synthetase-I, a mitochondrial enzyme responsible for elimination of ammonia through the urea cycle $[33,66]$. Since this enzyme system plays a significant role in the metabolism of excess ammonia, caution should be used when prescribing VPA to individuals with urea cycle defects [67-69]. The risk of hyperammonemia is greater when VPA is administered as part of anticonvulsant multi-therapy [33].

VPA-induced pancreatitis is often overlooked and underestimated by physicians. A worldwide literature review between 1979 and 2005 found only 90 patients in whom pancreatitis was associated with VPA [2]. Nevertheless, the increasing number of observations led the United States FDA to issue a black box warning on this drug in 2000 [70]. The mechanism of action of VPA-induced pancreatitis is considered idiosyncratic [71]. Pippenger et al. proposed a direct toxic effect on the pancreatic cell membrane by VPA via the production of free radicals that deplete superoxide dismutase (SOD), catalase (CAT), and glutathione peroxidase $[72,73]$. Although most cases are mild, VPA-induced pancreatitis can be severe and sometimes fatal. There is no correlation between serum VPA level and degree of injury; however, the combination of VPA-induced pancreatitis and hepatic injury has been positively correlated with increased morbidity and mortality [37].

There are numerous reports of valproate-associated coagulopathies. VPA has been reported to induce thrombocytopenia, abnormal platelet function, hypofibrinogemia, von Willebrandlike disorders, and a decrease in vitamin K-dependent factors [74-76]. Teich et al. reported a case of two patients who developed spontaneous bleeding while being treated with VPA. Laboratory findings attributed the coagulopathy to factor XIII deficiency which returned to baseline upon discontinuation of the drug [74]. Although this side effect has been known for approximately 30 years, there have been several disagreements with regards to the clinical relevance of VPA-associated changes in coagulation. Despite the controversies, reports of fatal subarachnoid hemorrhage, pulmonary hemorrhage, and pancytopenia from the therapeutic use of VPA have been documented. This has influenced many anesthesiologists and surgeons to stop VPA-treatment before surgery to avoid bleeding complications $[35,59,77,78]$.

\section{Are there any risk factors for VPA-toxicity?}

Bryant and Dreifuss reviewed all reported cases of fatal hepatotoxicity from VPA therapy that occurred between 1987 and 1993. They were able to identify 29 cases and found the following risk factors for mortality: age younger than 2 years, developmental delay, and concomitant metabolic disease $[32,79]$. The patients at greatest risk had complex neurological disorders requiring more than one antiepileptic drug [79]. Scheffner and Konig reported contradictory results in their review of VPA-hepatoxicity in Germany between 1980 and 1986. They found that only 2 of the 16 fatal cases occurred in children younger than 3 years of age; furthermore, only $26 \%$ of 91 published cases were younger than age 3 . They concluded that one cannot define a single high risk group and that physicians need to carefully monitor indices of liver dysfunction in patients who have no other alternatives to VPA therapy [44]. It is worthwhile noting that most cases of fatal VPA-associated hepatic 
failure have occurred during the first few months of initiating treatment; one study showed that the majority of cases occurred within the first 90 days after starting VPA therapy [80].

The majority of case reports of VPA-induced pancreatitis occurred in children, but this may be related to the high use of VPA in pediatric populations [2]. Gerstner et al. reviewed 16 cases of VPA-induced pancreatitis and found no significant differences in the concentrations of amylase and lipase between adults and children. Plaza et al. suggested that patients with end-stage renal disease are at increased risk for developing VPA-induced pancreatitis [39]. Werlin et al. charted 22 patients with VPA-associated pancreatitis over a 10 year period and found no relationship between the occurrence of pancreatitis and duration of VPA therapy, dosage, or use of other anticonvulsants [81].

\section{Clinically, how do these patients present after an acute VPA overdose?}

A patient who overdoses on VPA can present with a variety of signs and symptoms, including: ataxia, confusion, drowsiness, lethargy, and stupor $[3,70]$. Depending upon the degree of toxicity, these patients will present with variable gastrointestinal complaints, including: nausea, vomiting, abdominal pain, and diarrhea. Hypotension has been reported with massive VPA overdose. Anderson and Ritland reported the case of a 27-year-old female who presented with refractory hypotension after acutely ingesting an unknown amount of VPA. Despite aggressive resuscitation with fluids and treatment with vasopressors, the patient expired 3 days later [28]. Hypernatremia and metabolic acidosis with an elevated anion gap have been noted in patients with VPA toxicity $[25,26,82]$. In a multicenter review, Spiller et al. noted that $26 \%$ of patients with VPA ingestions and serum VPA levels $>450 \mu \mathrm{g} / \mathrm{mL}$ had an elevated anion gap (>15 mmol/L), while $6 \%$ of the patients had serum bicarbonate levels $<20 \mathrm{mEq} / \mathrm{L}$ [82].

\section{CASE CONTINUATION}

The patient's serum valproate level was $1016 \mu \mathrm{g} / \mathrm{mL}$. Additional chemistry results were: sodium $152 \mathrm{mEq} / \mathrm{L}$, potassium $4.5 \mathrm{mEql} / \mathrm{L}$, chloride $106 \mathrm{mEq} / \mathrm{L}$, bicarbonate $21 \mathrm{mEq} / \mathrm{L}$, BUN $7 \mathrm{mg} / \mathrm{dL}$, creatinine $1.3 \mathrm{mg} / \mathrm{dL}$, glucose $106 \mathrm{mg} / \mathrm{dL}$, AST $26 \mathrm{IU} / \mathrm{L}$, ALT $21 \mathrm{IU} / \mathrm{L}$, alkaline phosphatase $58 \mathrm{IU} / \mathrm{L}$, amylase $75 \mathrm{IU} / \mathrm{L}$, lipase $334 \mathrm{IU} / \mathrm{L}$, and serum osmolality $307 \mathrm{mOs} / \mathrm{kg}$. Coagulation studies were PT $11.4 \mathrm{sec}$ (INR 1.09) and PTT $25.2 \mathrm{sec}$. Initial serum ammonia level was $371 \mu \mathrm{mol} / \mathrm{L}$. A serum drug screen was negative for salicylates, acetaminophen, cyclic antidepressants and ethanol, as was the urine drugs-of-abuse screen using the Abbott's Microparticle Enzyme Immunoassay (MEIA) for cocaine, amphetamines, marijuana, barbiturates, benzodiazepines, opiates, and phencyclidine. The patient was subsequently admitted to the ICU for continuous monitoring and observation.

\section{How do you manage patients with valproic acid toxicity?}

Managing VPA intoxicated patients involves close attention to airway support, appropriate decontamination, supportive treatment, and closely monitoring specific labs. Because VPA affects the concentration of GABA in the brain, patients with an overdose may present with profound CNS depression, and physicians should have a low threshold for aggressive airway protection. In one study, endotracheal intubation was required in $63 \%$ of patients with serum valproate levels of $450 \mu \mathrm{g} / \mathrm{mL}$ or higher [83].

Gastric decontamination includes an initial dose of activated charcoal in accordance to ACCT/EAPCC guidelines [84]. Whole bowel irrigation is recommended for ingestions of enteric-coated or sustained-release tablets or with concomitant drug ingestions that may delay gut motility [23]. The use of multiple dose activated charcoal was also shown to be helpful in preventing delayed absorption of VPA; however, unlike its effect on other antiepileptic agents such as phenytoin or carbamazepine, multi-dose charcoal had no effect in enhancing the elimination half-life [22].

Although the absolute serum VPA value does not always correlate with toxicity, serial drug levels should be followed to determine trends in the pharmacokinetics. There are reports of patients presenting in a coma with normal serum VPA concentrations; this is possibly due to the presence of unmeasured metabolites such as 2-en-VPA and 4-en-VPA or due to hyperammonemia [28,33,42]. Ammonia levels, therefore, should be obtained in all cases of suspected VPA toxicity. Serum amylase, lipase, liver function profile, electrolytes, glucose, and coagulation parameters should be quantified. Signs and symptoms associated with fulminant hepatic failure should be closely monitored because of numerous reports of fatalities from VPA-associated hepatic failure $[43,80,85]$. Bell et al. reported a case of a 23-year-old woman who developed fulminant hepatic failure secondary to VPA toxicity that required orthotopic liver transplantation [85]. The patient recovered.

There are currently no antidotal therapies available to reverse the clinical effects of VPA. Acute VPA intoxications can sometime present with findings similar to an opioid toxidrome; hence, the use of naloxone has been suggested $[8,86]$. Steiman et al. described a case where naloxone was administered with success after an acute VPA ingestion [86]. However, in some other cases, naloxone showed no effect [87]. Since naloxone produced no adverse reactions in VPA toxicity, its use is often indicated in a comatose patient with unknown etiology.

Because of its role in restoring $\beta$-oxidation metabolism in the mitochondrial cells of the liver, some authors have suggested a role for carnitine supplementation. Ishikura et al. and Murakami et al. each presented a case in which the administration of L-carnitine increased the metabolism of VPA via $\beta$-oxidation by passing the $\omega$-oxidation and the production of the toxic metabolite $[36,40]$. Another study showed that supplementing L-carnitine in epileptic children with two or more risk factors for developing functional carnitine deficiency may be beneficial. Some of those children showed signs of clinical and neurological improvements when given carnitine supplementation [88]. Based on the numerous case reports, the 1996 Pediatric Neurology Advisory Committee includes the use intravenous L-carnitine in their guidelines for all cases of valproate-induced hepatotoxicity and for severe valproate overdose [32]. In addition, they 
strongly recommend oral supplementation for patients at risk of developing carnitine deficiency, symptomatic VPA-associated hyperammonemia, children younger than 2 years of age with complex neurologic disorder, and patients who have multiple risk factors for hepatotoxicity [32,53]. Interestingly, there is no convincing evidence that L-carnitine supplementation for acute VPA overdose or VPA-induced hyperammonemia and hepatotoxicity changes clinical outcome. In the case described by Ishikura et al., a 16-month-old baby boy regained consciousness only after serum VPA levels reached therapeutic levels, despite being on a 4 day course of carnitine [36]. Laub et al. reported a case of 3-year-old child who developed fatal hepatoxicity while being treated with VPA; he died 17 days later despite being on carnitine supplementation [89]. Nevertheless, given the lack of available data and its relatively safe profile, the use of L-carnitine remains a reasonable option for patients with acute overdose or VPA-induced hepatoxicity and hyperammonemia [38].

With acute VPA overdose or in patients with hyperammonemia, the dosing for L-carnitine is $150-500 \mathrm{mg} / \mathrm{kg} /$ day orally or intravenously up to a total of $3 \mathrm{~g} /$ day in 3 divided doses [8]. There are limited studies to determine the appropriate time frame for carnitine supplementation. One study showed reversal of hyperammonemia and hepatic injury by supplementing Lcarnitine for four weeks in children on chronic VPA dosing [41]. For normal healthy individuals started on VPA, the 1996 Pediatric Neurology Advisory Committee recommends oral administration of L-carnitine of $50-100 \mathrm{mg} / \mathrm{kg} /$ day up to $2 \mathrm{~g} /$ day in divided doses during the course of treatment or during an acute overdose with VPA [32].

\section{Is L-carnitine safe?}

There have been a limited number of studies evaluating the safety of L-carnitine. Supplementing L-carnitine is considered safe because it is endogenously produced and found naturally in our diet. One study reviewed the safety of L-carnitine in patients with acute VPA ingestion reported to the poison center during a 3 year period. They found that L-carnitine was administered to 251 patients without any adverse effects [38]. The limitation of this study is that the authors were examining only two adverse reactions: anaphylaxis and hypotension. Hathcock and Shao reviewed a number of published human clinical trial data examining the risk and safety of chronic L-carnitine supplementation at different prescribed doses. They found that the highest observed safe level (OSL) for L-carnitine was $2 \mathrm{~g} /$ day. There are clinical trials that administered 4-6 grams of L-carnitine without any adverse effects; however, the lack of sufficient data cannot fully support bumping the OSL to such a high dose for chronic supplementation [49].

With excessive ingestion, carnitine may have limited absorption through the gastrointestinal tract. In several observational studies, it was noted that individuals on high carnitine diets $(>6$ grams) had approximately $5-15 \%$ absorption, while those on a low load diet of carnitine $(<1 \mathrm{~g})$, such as a vegan diet, had greater than $75 \%$ of the carnitine absorbed $[52,90,91]$. This apparent limit in absorptive capacity may limit the clinical effects on an overdose of oral L-carnitine.

\section{Can we use extracorporeal drug removal in these patients?}

Although the management of VPA-toxic patients is largely supportive, there are certain instances when the use of extracorporeal therapy may be pivotal for the survival of the patient.

VPA is poorly water soluble, but it has a low volume of distribution $(0.1-0.5 \mathrm{~L} / \mathrm{kg})$ and a low molecular weight, allowing it to be effectively removed from circulation by dialysis [26]. During VPA overdose, protein binding sites saturate and lead to an increased amount of free VPA. Kane et al. reported a case of valproic acid overdose that was effectively treated with highflux hemodialyis, reducing the serum half- life of VPA from 23.41 hours to 2.74 hours [26]. In another case report, high-flux hemodialysis was instituted in a 24 -year-old male patient who was comatose after ingesting about 30 grams of VPA and developed respiratory instability and severe cardiovascular shock. After two courses of hemodialysis, the patient began to show clinical and neurological improvement [92]. The authors concluded that high-flux hemodialysis was safe and effective in removing VPA. Because of limited data, there are no clear guidelines for dialysis in VPA toxicity. Some authors suggest termination of dialysis once the patient begins to show neurological improvement and when VPA levels decline to therapeutic range $(50-100 \mu \mathrm{g} / \mathrm{mL})$ or less [93]. Extreme caution should be advised when attempting to aggressively lower the serum VPA concentration in a patient taking the drug for a seizure disorder, since seizures may occur once levels drop below therapeutic values.

The pharmacokinetic properties of VPA suggest that it is effectively removed with charcoal hemoperfusion; however, in one report, hemoperfusion did not accelerate valproic acid elimination in a patient with a peak concentration of $1080 \mu \mathrm{g} / \mathrm{mL}$ [46]. Several reports suggest that the combination of hemodialysis and hemoperfusion (HD/HP) is highly effective in reducing serum VPA levels and minimizing toxicity. Tank and Palmer used the combination of HD/HP in a patient with peak plasma concentration of $1262 \mu \mathrm{g} / \mathrm{mL}$, and they found that the elimination half-life of VPA reduced from 13 hrs to 1.7 hrs during the procedure [24]. Charcoal hemoperfusion, however, is not available in many medical centers and can produce severe side effects, including: coagulopathy, electrolyte disturbances, and hypothermia. For this reason, high-flux hemodialysis is favored for the management of severe VPA poisonings [26,92,94].

Currently, there is insufficient clinical experience to propose a threshold value for initiating extracorporeal removal. In a multicenter case series, Spiller et al. found that a peak VPA concentration of $850 \mu \mathrm{g} / \mathrm{mL}$ was sufficient to warrant hemodialysis [82]. In the case presented by Keilstein et al., the authors concluded performing hemodialysis solely on the clinical grounds and not drug levels [92]. Since serum VPA concentrations do not necessarily correlate well with clinical symptoms, we recommend extra-corporeal therapy for those patients who present with hemodynamic and respiratory instability after overdosing on VPA. 


\section{CASE CONCLUSION}

The patient was treated with whole bowel irrigation in conjunction with multi-dose activated charcoal, administered every 4 hours. He was also started on 1 gram of L-carnitine intravenously every $8 \mathrm{hrs}$. On the second day of admission, his repeat serum valproate level was $78 \mu \mathrm{g} / \mathrm{ml}$ and his serum ammonia concentration decreased to $38 \mu \mathrm{mol} / \mathrm{L}$. Later that afternoon, he was extubated without any clinical regression. He continued to do well throughout the evening without displaying any neurological problems or clinical signs of hepatotoxicity. The next day, the patient was medically cleared for psychiatric evaluation.

The authors have no potential financial conflicts of interest to report.

\section{REFERENCES}

1. Henry, T.R., The history of valproate in clinical neuroscience. Psychopharmacol Bull 2003;37 Suppl 2:5-16.

2. Gerstner, T., et al., Valproic acid-induced pancreatitis: 16 new cases and a review of the literature. J Gastroenterol 2007; 42(1):39-48.

3. Doyon, S., Anticonvulsants. 8th ed. Goldfrank's Toxicologic Emergencies, ed. N. Flomenbaum, Howland, MA, Goldfrank, LR, Lewin, NA, Hoffman, RS, \& Nelson, LS. 2006, New York: McGraw-Hill. 731-745.

4. Brubacher, J.R., P. Dahghani, and D. McKnight, Delayed toxicity following ingestion of enteric-coated divalproex sodium (Epival). J Emerg Med 1999; 17(3):463-467.

5. Routy, J.P., Valproic acid: a potential role in treating latent HIV infection. Lancet 2005;366(9485):523-524.

6. Schwartz, C., et al., Valproic acid induces non-apoptotic cell death mechanisms in multiple myeloma cell lines. Int $J$ Oncol 2007;30(3):573-582.

7. Valentini, A., et al., Valproic Acid Induces Apoptosis, $\mathrm{p}(16 \mathrm{INK} 4 \mathrm{~A})$ Upregulation and Sensitization to Chemotherapy in Human Melanoma Cells. Cancer Biol Ther 2007;6(2).

8. Sztajnkrycer, M.D., Valproic acid toxicity: overview and management. J Toxicol Clin Toxicol 2002;40(6):789-801.

9. Litovitz, T.L., et al., 1990 annual report of the American Association of Poison Control Centers National Data Collection System. Am J Emerg Med 19919(5):461-509.

10. Litovitz, T.L., et al., 1995 annual report of the American Association of Poison Control Centers Toxic Exposure Surveillance System. Am J Emerg Med 1996;14(5):487-537.

11. Litovitz, T.L., et al., 2000 Annual report of the American Association of Poison Control Centers Toxic Exposure Surveillance System. Am J Emerg Med 2001; 19(5):337-395.

12. Hariton, C., et al., Distribution of sodium valproate and GABA metabolism in CNS of the rat. Biopharm Drug Dispos 1984;5(4):409-414.

13. Johannessen, C.U., Mechanisms of action of valproate: a commentatory. Neurochem Int 2000;37(2-3):103-110.
14. Gean, P.W., et al., Valproic acid suppresses the synaptic response mediated by the NMDA receptors in rat amygdalar slices. Brain Res Bull 1994;33(3):333-336.

15. Griffith, W.H. and L. Taylor, Sodium valproate decreases synaptic potentiation and epileptiform activity in hippocampus. Brain Res 1988474(1):155-164.

16. Zeise, M.L., S. Kasparow, and W. Zieglgansberger, Valproate suppresses N-methyl-D-aspartate-evoked, transient depolarizations in the rat neocortex in vitro. Brain Res 1991;544(2):345-348.

17. McLean, M.J. and R.L. Macdonald, Sodium valproate, but not ethosuximide, produces use- and voltage-dependent limitation of high frequency repetitive firing of action potentials of mouse central neurons in cell culture. J Pharmacol Exp Ther 1986; 237(3):1001-1011.

18. Albus, H. and R. Williamson, Electrophysiologic analysis of the actions of valproate on pyramidal neurons in the rat hippocampal slice. Epilepsia 1998; 39(2):124-139.

19. Lheureux, P.E., et al., Science review: carnitine in the treatment of valproic acid-induced toxicity - what is the evidence? Crit Care 2005;9(5):431-440.

20. Klotz, U., Bioavailability of a slow-release preparation of valproic acid under steady-state conditions. Int J Clin Pharmacol Ther Toxicol 1982;20(1):24-26.

21. Klotz, U. and K.H. Antonin, Pharmacokinetics and bioavailability of sodium valproate. Clin Pharmacol Ther 1977;21(6):736-743.

22. al-Shareef, A., et al., The effect of repeated-dose activated charcoal on the pharmacokinetics of sodium valproate in healthy volunteers. Br J Clin Pharmacol 1997;43(1):109-111.

23. Graudins, A. and C.K. Aaron, Delayed peak serum valproic acid in massive divalproex overdose-treatment with charcoal hemoperfusion. J Toxicol Clin Toxicol 1996;34(3):335-341.

24. Tank, J.E. and B.F. Palmer, Simultaneous "in series" hemodialysis and hemoperfusion in the management of valproic acid overdose. Am J Kidney Dis 1993;22(2):341-344.

25. Franssen, E.J., et al., Valproic acid toxicokinetics: serial hemodialysis and hemoperfusion. Ther Drug Monit 1999;21(3):289-292.

26. Kane, S.L., et al., High-flux hemodialysis without hemoperfusion is effective in acute valproic acid overdose. Ann Pharmacother 2000;34(10):1146-1151.

27. Brewster, D. and N.C. Muir, Valproate plasma protein binding in the uremic condition. Clin Pharmacol Ther 1980;27(1):76-82.

28. Andersen, G.O. and S. Ritland, Life threatening intoxication with sodium valproate. J Toxicol Clin Toxicol 1995;33(3):279-284.

29. Nau, H. and W. Loscher, Valproic acid and metabolites: pharmacological and toxicological studies. Epilepsia 1984;25 Suppl 1:S14-S22.

30. Stahl, J., Studies of the blood ammonia in liver disease. Its diagnostic, prognostic, and therapeutic significance. Ann Intern Med 1963;58:1-24. 
31. Sugimoto, T., et al., Valproate metabolites in high-dose valproate plus phenytoin therapy. Epilepsia 1996; 37(12):1200-1203.

32. De Vivo, D.C., et al., L-carnitine supplementation in childhood epilepsy: current perspectives. Epilepsia

1998;39(11):1216-1225.

33. Duarte, J., et al., Valproate-induced coma: case report and literature review. Ann Pharmacother 1993;27(5):582-583.

34. Eyer, F., et al., Acute valproate poisoning: pharmacokinetics, alteration in fatty acid metabolism, and changes during therapy. J Clin Psychopharmacol 2005; 25(4):376-380.

35. Gerstner, T., et al., Valproic acid induced encephalopathy19 new cases in Germany from 1994 to 2003-a side effect associated to VPA-therapy not only in young children. Seizure

2006; 15(6):443-448.

36. Ishikura, H., et al., Valproic acid overdose and L-carnitine therapy. J Anal Toxicol 1996;20(1):55-58.

37. Koenig, S.A., et al., Valproic acid-induced hepatopathy: nine new fatalities in Germany from 1994 to 2003. Epilepsia 2006;47(12):2027-2031.

38. LoVecchio, F., J. Shriki, and R. Samaddar, L-carnitine was safely administered in the setting of valproate toxicity. Am J Emerg Med 2005;23(3):321-322.

39. Moreiras Plaza, M., et al., On the toxicity of valproic-acid. Clin Nephrol 1999 51(3):187-189.

40. Murakami K., et al., Effect of L-carnitine supplementation on acute valproate intoxication. Epilepsia 1996;37(7):687-689.

41. Ohtani, Y., F. Endo, and I. Matsuda, Carnitine deficiency and hyperammonemia associated with valproic acid therapy. J Pediatr 1982;101(5):782-785.

42. Rawat, S., W.J. Borkowski, Jr., and H.M. Swick, Valproic acid and secondary hyperammonemia. Neurology

1981;31(9):1173-1174.

43. Scheffner, D., Fatal liver failure in children on valproate.

Lancet 1986;2(8505):511.

44. Scheffner, D. and S. Konig, Valproate hepatotoxicity.

Lancet 1987;1(8529):389-390.

45. Stadler, D.D., et al., Effect of long-term valproic acid administration on the efficiency of carnitine reabsorption in humans. Metabolism 1999;48(1):74-79.

46. van der Merwe, A.C., et al., Sodium valproate poisoning. A case report. $S$ Afr Med J 1985;67(18):735-736.

47. Verrotti, A., et al., Carnitine deficiency and hyperammonemia in children receiving valproic acid with and without other anticonvulsant drugs. Int J Clin Lab Res 1999;29(1):36-40.

48. Warter, J.M., et al., The renal origin of sodium valproateinduced hyperammonemia in fasting humans. Neurology 198333(9):1136-1140.

49. Hathcock, J.N. and A. Shao, Risk assessment for carnitine. Regul Toxicol Pharmacol 2006;46(1):23-28.

50. Steiber, A., J. Kerner, and C.L. Hoppel, Carnitine: a nutritional, biosynthetic, and functional perspective. Mol Aspects Med; 2004;25(5-6):455-473.

51. Scaglia, F., Carnitine Deficiency, in Emedicine, C.J. Renner, Editor. 2006; WebMD.
52. Sahajwalla, C.G., et al., Multiple-dose pharmacokinetics and bioequivalence of L-carnitine 330-mg tablet versus 1-g chewable tablet versus enteral solution in healthy adult male volunteers. J Pharm Sci 1995;84(5):627-633.

53. Raskind, J.Y. and G.M. El-Chaar, The role of carnitine supplementation during valproic acid therapy. Ann Pharmacother 2000;34(5):630-638.

54. Carter, A.L., T.O. Abney, and D.F. Lapp, Biosynthesis and metabolism of carnitine. J Child Neurol 1995;10 Suppl 2:S3-S7.

55. Rebouche, C.J., Renal handling of carnitine in experimental vitamin C deficiency. Metabolism, 1995;44(12):1639-1643.

56. Okamura, N., et al., Involvement of recognition and interaction of carnitine transporter in the decrease of L-carnitine concentration induced by pivalic acid and valproic acid. Pharm Res 2006;23(8):1729-1735.

57. Roe, C.R., et al., Carnitine homeostasis in the organic acidurias. Prog Clin Biol Res 1990;321:383-402.

58. Dupuis, R.E., S.N. Lichtman, and G.M. Pollack, Acute valproic acid overdose. Clinical course and pharmacokinetic disposition of valproic acid and metabolites. Drug Saf 1990;5(1):65-71.

59. Rajantie, J., M. Kajosaari, and V. Ylitalo, Fatal pancytopenia during high-dose valproate monotherapy. Eur J Pediatr 1992; 151(8):619.

60. Coulter, D.L., Carnitine deficiency: a possible mechanism for valproate hepatotoxicity. Lancet;1984. 1(8378):689.

61. Kuhara, T., et al., Markedly increased omega-oxidation of valproate in fulminant hepatic failure. Epilepsia

1990;31(2):214-217.

62. Kesterson, J.W., G.R. Granneman, and J.M. Machinist, The hepatotoxicity of valproic acid and its metabolites in rats. I. Toxicologic, biochemical and histopathologic studies.

Hepatology 1984;4(6):1143-1152.

63. Rettie, A.E., et al., Cytochrome P-450—catalyzed formation of delta 4-VPA, a toxic metabolite of valproic acid. Science 1987;235(4791):890-893.

64. Brusilow, S.W., Hyperammonemic encephalopathy. Medicine (Baltimore) 2002; 81(3):240-249.

65. Sherlock, S., Fulminant hepatic failure. Adv Intern Med 1993;38:245-267.

66. Cotariu, D. and J.L. Zaidman, Valproic acid and the liver. Clin Chem 1988;34(5):890-897.

67. Thakur, V., et al., Fatal cerebral edema from late-onset ornithine transcarbamylase deficiency in a juvenile male patient receiving valproic acid. Pediatr Crit Care Med 2006;7(3):273-276.

68. Hjelm, M., et al., Evidence of inherited urea cycle defect in a case of fatal valproate toxicity. Br Med J (Clin Res Ed) 1986;292(6512):23-24.

69. Hjelm, M., et al., Valproate-induced inhibition of urea synthesis and hyperammonaemia in healthy subjects. Lancet 1986;2(8511):859.

70. Pellock, J.M., et al., Acute pancreatitis coincident with valproate use: a critical review. Epilepsia 2002;43(11):1421-1424.

71. Asconape, J.J., et al., Valproate-associated pancreatitis. Epilepsia 1993:34(1):177-183. 
72. Pippenger CE, M.X., Rothner AD, Cruse RP, Erenberg G, and Solano R., Free radical scavenging enzyme activity profiles in risk assessment of idiosyncratic drug reactions. Idiosyncratic reactions to valproate: clinical risk patterns and mechanisms of toxicity, ed. L.R.a.P. JK. 1991; New York: Raven Press Ltd. 75-88.

73. Underwood, T.W. and C.B. Frye, Drug-induced pancreatitis. Clin Pharm 1993 12(6):440-448.

74. Teich, M., et al., Factor XIII deficiency associated with valproate treatment. Epilepsia 2004;45(2):187-189.

75. Gerstner, T., et al., Valproate-associated coagulopathies are frequent and variable in children. Epilepsia

2006;47(7):1136-1143.

76. Gidal, B., et al., Valproate-mediated disturbances of hemostasis: relationship to dose and plasma concentration. Neurology 1994;44(8):1418-1422.

77. Sleiman, C., et al., Fatal pulmonary hemorrhage during high-dose valproate monotherapy. Chest 2000;117(2):613.

78. Winter, S.L., et al., Perioperative blood loss: the effect of valproate. Pediatr Neurol 1996;15(1):19-22.

79. Bryant, A.E., 3rd and F.E. Dreifuss, Valproic acid hepatic fatalities. III. U.S. experience since 1986. Neurology 1996;46(2):465-469.

80. Dreifuss, F.E., et al., Valproic acid hepatic fatalities. II. US experience since 1984. Neurology 1989;39(2 Pt 1):201-207.

81. Werlin, S.L. and D.L. Fish, The spectrum of valproic acidassociated pancreatitis. Pediatrics 2006;118(4):1660-1663.

82. Spiller, H.A., et al., Multicenter case series of valproic acid ingestion: serum concentrations and toxicity. J Toxicol Clin Toxicol 2000;38(7):755-760.

83. Beauchamp, J., Oslon, K., Valproic Acid Overdose: A Retrospective Study Comparing Serum Drug Levels And The Incidence Of Adverse Outcomes. J Toxicol Clin Toxicol 1999;37:637-638.
84. Chyka, P.A. and D. Seger, Position statement: single-dose activated charcoal. American Academy of Clinical Toxicology; European Association of Poisons Centres and Clinical Toxicologists. J Toxicol Clin Toxicol 1997;35(7):721-741.

85. Bell, E.A., et al., Treatment of valproic acid-associated hepatic failure with orthotopic liver transplantation. Ann Pharmacother 1992;26(1):18-21.

86. Steiman, G.S., R.W. Woerpel, and E.S. Sherard, Jr., Treatment of accidental sodium valproate overdose with an opiate antagonist. Ann Neurol 1979;6(3):274.

87. Mortensen, P.B., et al., Acute valproate intoxication: biochemical investigations and hemodialysis treatment. Int J Clin Pharmacol Ther Toxicol 1983;21(2):64-68.

88. Coulter, D.L., Carnitine and anticonvulsant drugs. L-carnitine in the pharmacotherapy of epilepsy 1994;9-15.

89. Laub, M.C., I. Paetzke-Brunner, and G. Jaeger, Serum carnitine during valproic acid therapy. Epilepsia

1986;27(5):559-562.

90. Rebouche, C.J. and C.A. Chenard, Metabolic fate of dietary carnitine in human adults: identification and quantification of urinary and fecal metabolites. J Nutr 1991;121(4):539-546.

91. Harper, P., C.E. Elwin, and G. Cederblad, Pharmacokinetics of bolus intravenous and oral doses of L-carnitine in healthy subjects. Eur J Clin Pharmacol 1988;35(1):69-75.

92. Kielstein, J.T., et al., Efficiency of high-flux hemodialysis in the treatment of valproic acid intoxication. J Toxicol Clin Toxicol 2003;41(6):873-876.

93. Singh, S.M., et al., Extracorporeal management of valproic acid overdose: a large regional experience. J Nephrol

2004; 17(1):43-49.

94. Hicks, L.K. and P.A. McFarlane, Valproic acid overdose and haemodialysis. Nephrol Dial Transplant, 2001;16(7):1483-1486. 